\title{
Picomolar to Micromolar: Elucidating the Role of Distal Mutations in HIV-1 Protease in Conferring Drug Resistance
}

\author{
Mina Henes, ${ }^{\dagger, \S}$ Gordon J. Lockbaum, ${ }^{\dagger, \S ®}$ Klajdi Kosovrasti, ${ }^{\dagger}$ Florian Leidner, $^{\dagger}$ Gily S. Nachum, ${ }^{\dagger}$ \\ Ellen A. Nalivaika, ${ }^{\dagger}$ Sook-Kyung Lee, ${ }^{\ddagger}$ Ean Spielvogel, ${ }^{\ddagger}$ Shuntai Zhou, ${ }^{\ddagger}$ Ronald Swanstrom, \\ Daniel N.A. Bolon, ${ }^{\dagger}$ Nese Kurt Yilmaz, ${ }^{*} \dagger$ and Celia A. Schiffer ${ }^{*}, \dagger$ \\ ${ }^{\dagger}$ Department of Biochemistry and Molecular Pharmacology, University of Massachusetts Medical School, Worcester, Massachusetts \\ 01605, United States \\ ${ }^{\ddagger}$ Department of Biochemistry and Biophysics and the UNC Center for AIDS Research, University of North Carolina at Chapel Hill, \\ Chapel Hill, North Carolina 27599, United States
}

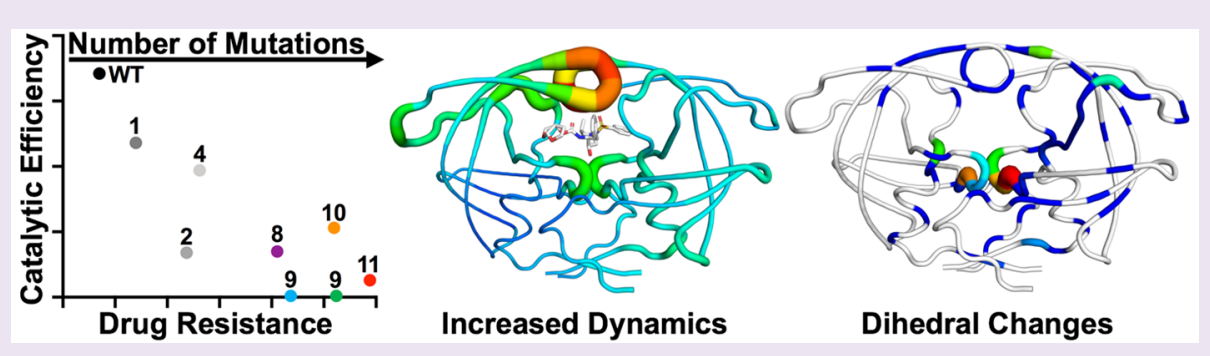

ABSTRACT: Drug resistance continues to be a growing global problem. The efficacy of small molecule inhibitors is threatened by pools of genetic diversity in all systems, including antibacterials, antifungals, cancer therapeutics, and antivirals. Resistant variants often include combinations of active site mutations and distal "secondary" mutations, which are thought to compensate for losses in enzymatic activity. HIV-1 protease is the ideal model system to investigate these combinations and underlying molecular mechanisms of resistance. Darunavir (DRV) binds wild-type (WT) HIV-1 protease with a potency of $<5$ pM, but we have identified a protease variant that loses potency to DRV 150000 -fold, with 11 mutations in and outside the active site. To elucidate the roles of these mutations in DRV resistance, we used a multidisciplinary approach, combining enzymatic assays, crystallography, and molecular dynamics simulations. Analysis of protease variants with 1, 2, 4, 8, 9, 10, and 11 mutations showed that the primary active site mutations caused $\sim 50$-fold loss in potency ( 2 mutations), while distal mutations outside the active site further decreased DRV potency from $13 \mathrm{nM}$ ( 8 mutations) to $0.76 \mu \mathrm{M}$ (11 mutations). Crystal structures and simulations revealed that distal mutations induce subtle changes that are dynamically propagated through the protease. Our results reveal that changes remote from the active site directly and dramatically impact the potency of the inhibitor. Moreover, we find interdependent effects of mutations in conferring high levels of resistance. These mechanisms of resistance are likely applicable to many other quickly evolving drug targets, and the insights may have implications for the design of more robust inhibitors.

$\mathrm{D}$ rug resistance is a growing global problem. ${ }^{1,2}$ The efficacy of current drugs is threatened by pools of genetic diversity in many therapeutic targets, including antibacterials, antifungals, cancer therapeutics, and antivirals. ${ }^{3}$ Specifically, rapid evolution in viruses can cause emergence of variants that are not effectively inhibited by small molecule inhibitors. Human immunodeficiency virus (HIV) is capable of accumulating a large number of mutations in response to antiretroviral therapy. ${ }^{4,5}$

The viral enzymes coded by the HIV genome, including HIV-1 protease, have been targets of extensive drug development efforts. HIV-1 entry, nucleoside reverse transcriptase, non-nucleoside reverse transcriptase, integrase, and protease inhibitors are used in combination to curb the emergence of drug resistance. Resistance can develop quickly against a single agent, with mutations resulting from the high infidelity of the reverse transcriptase ${ }^{6-8}$ and the high recombination rate of HIV. ${ }^{9,10}$ Mutations may also arise from patient noncompliance due to a number of factors, such as adverse side effects and high pill burden. ${ }^{11} \mathrm{HIV}-1$ protease can tolerate a large number of mutations, with 45 out of 99 amino acid positions able to mutate while retaining adequate enzymatic activity to allow replication. ${ }^{12}$ Viral variants from patients who failed therapy and in vitro selection experiments often contain complex combinations of multiple mutations. Especially with potent inhibitors, a single mutation is not sufficient and many

Special Issue: Proteolytic Regulation of Cellular Physiology

Received: May 8, 2019

Accepted: July 30, 2019

Published: July 30, 2019 

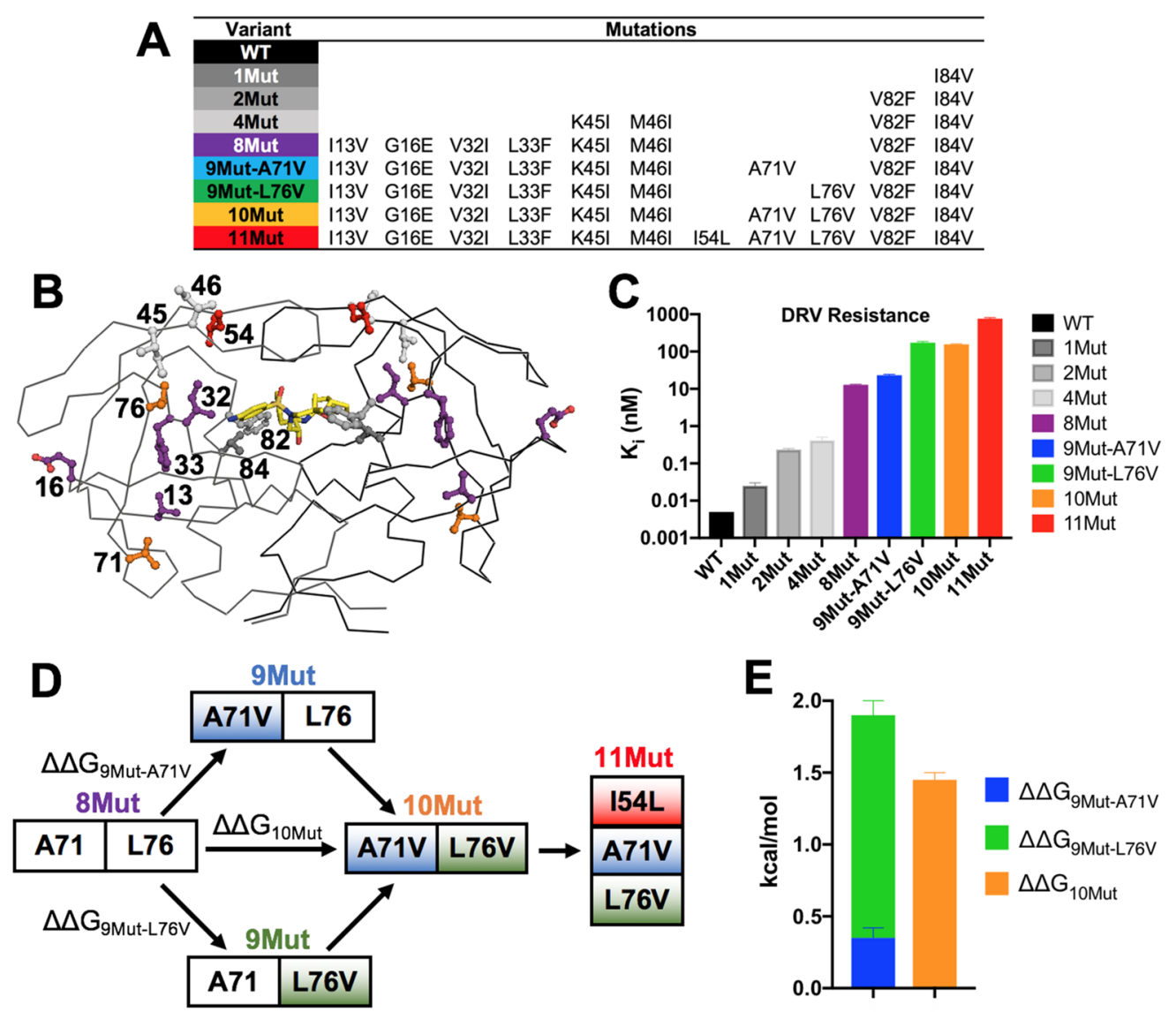

Figure 1. (A, B) Resistance mutations in the variants, displayed on HIV-1 protease structure in panel B, with side chain of residues mutated shown as ball and stick and colored to match the variant added to in panels A and C. DRV depicted as yellow sticks. Mutations V32I, V82F, and I84V are classified as proximal, while all other mutations are distal. (C) Inhibition constant, $K_{\mathrm{i}}$, of each variant against DRV. Resistance increases as mutations accumulate. (D) Double mutant cycle of distal mutations A71V and L76V going from 8Mut to 10Mut variant with 12-fold higher resistance. The additional I54L mutation (11Mut) further increases resistance 5-fold. (E) Mutations are interdependent $\left(\Delta \Delta G_{9 \mathrm{Mut}-\mathrm{A} 71 \mathrm{~V}}+\right.$ $\left.\Delta \Delta G_{9 \text { Mut-L76 }} \neq \Delta \Delta G_{10 \text { Mut }}\right)$.

mutations need to accumulate to confer appreciable levels of resistance. Under drug pressure, resistance and compensatory mutations accumulate in the Gag and Gag-Pol viral polyproteins, resulting in often unpredictable and complex mechanisms of resistance. ${ }^{13,14}$ Given the prevalence and complexity of these mutations and underlying mechanisms, HIV-1 has served as a model system to identify, analyze, and characterize drug resistance.

Darunavir (DRV) is the latest and most potent FDAapproved HIV-1 protease inhibitor. ${ }^{15}$ The potency of DRV is due to several factors, including a number of crucial hydrogen bonds with the protease backbone, conservation of the watermediated hydrogen bonding network with the flaps, and extensive van der Waals (vdW) contacts with active site residues. $^{16,17}$ Moreover, DRV fits well within the substrate envelope (the consensus volume occupied by the natural substrates ${ }^{18}$ ), which explains its low susceptibility to resistance. ${ }^{19-21}$ DRV can effectively inhibit variants with common single primary resistance mutations such as $\mathrm{I} 84 \mathrm{~V}$ and $\mathrm{I} 50 \mathrm{~V} / \mathrm{A} 71 \mathrm{~V} .^{22}$ However, high levels of resistance are reached as mutations accumulate, both within and outside the active site of HIV-1 protease.

The combinations of mutations in $\mathrm{HIV}-1$ protease can utilize complex mechanisms of resistance to reach significant levels of resistance. The primary mutations proximal to the active site are thought to directly confer drug resistance while distal mutations are considered compensatory and restore catalytic function. ${ }^{23}$ However, distal mutations can also weaken inhibitor binding, as has been reported for not only HIV-1 protease but other drug targets as well. ${ }^{24-27}$ Molecular mechanisms such as reducing the coupled motions of the protease and inhibitor ${ }^{28}$ and altering protein intramolecular hydrogen bonds ${ }^{29}$ have all been suggested in resistance mechanisms involving mutations distal to the inhibitor binding site. We have previously shown that cross-correlations between protein and inhibitor fluctuations distinguish tight binders from weak binders in both HIV and HCV protease. ${ }^{29-31}$ In addition to weakening inhibitor binding, mutations can alter the dynamic ensemble of the protease-inhibitor complex and the balance between inhibition and catalytic activity. However, changes to the structure and dynamic ensemble along a resistance pathway, molecular mechanisms of resistance due to combinations of mutations, and the role of distal mutations in conferring resistance versus restoring catalytic activity are not well understood.

Here we investigate highly mutated and highly DRV resistant variants of HIV-1 protease to understand the role of distal mutations and uncover underlying mechanisms of resistance. While DRV inhibits wild-type HIV-1 protease with picomolar inhibition constant, a combination of 11 mutations ( 3 active site and 8 distal) confers near micromolar resistance to DRV. All of these mutations have been observed 
in patient isolates, although not in this specific combination. $^{32,33}$ Our inhibition assays show that the active site mutations alone do not account for this severe loss in affinity to DRV. We generated protease variants with subsets of these 11 mutations $(1,2,4,8$, and 10 mutant variants) and found increased resistance with accumulation of mutations. We also tease out the effect of two individual distal mutations on the viral evolution pathway from 8 to 10 mutant variants, through double mutant cycle analysis of the two possible intermediate 9 mutant variants. Using high-resolution crystal structures, enzymatic and inhibition assays, and molecular dynamics simulations, our findings support the role of distal mutations in conferring drug resistance. In addition to restoring catalytic activity, distal mutations contribute to drug resistance through interdependent, dynamic processes propagated throughout the protease.

\section{RESULTS}

Viral Passaging under Drug Pressure Selected for Highly Mutated Variants. Viral passaging experiments were performed to elucidate drug resistance in HIV-1 protease under increasing DRV selective pressure and identified 2 highly abundant variants with 8 and 10 mutations. ${ }^{34}$ At the highest drug concentration, an additional mutation was selected, leading to a variant with 11 total mutations (11Mut) [Figure $1 A, B]$. Sequencing from early time points indicated that I84V (1Mut) was the first fixed mutation. A variant with both V82F and $\mathrm{I} 84 \mathrm{~V}$ (2Mut) was generated to investigate primary active site mutations. A midpoint variant between 2 Mut and $8 \mathrm{Mut}$ was chosen to include 2 active site and 2 flap mutations (I84V, V82F, K45I, and M46I; 4Mut variant). Addition of 4 more mutations (I13V, G16E, V32I, and I33F) to 4Mut yielded the 8 Mut variant that was highly abundant in the viral passaging at higher DRV concentrations. With increased DRV pressure, the next highly abundant variant included additional A71V and L76V mutations (10Mut). The possible 9 mutant intermediate variants en route to $10 \mathrm{Mut}$ from $8 \mathrm{Mut}$ were not observed in viral passaging. To understand the role of the additional A71V and L76V mutations individually, 9Mut-A71V and 9Mut-L76V variants were generated. Lastly, the addition of I54L mutation onto $10 \mathrm{Mut}$ created the $11 \mathrm{Mut}$ variant. For all these 8 variants [Figure 1A] and wild-type protease, the catalytic activity, DRV inhibition constant, and high-resolution crystal structure were determined, and $\mathrm{MD}$ simulations were performed to elucidate the molecular mechanisms of resistance.

Resistance Mutations Are Selected to Maintain Catalytic Activity. To determine the effects of mutation accumulation on protease catalytic activity, an established enzyme assay for cleaving the natural MA/CA site was used to measure the Michaelis-Menten constant $\left(K_{\mathrm{M}}\right)$ and turnover rate $\left(k_{\text {cat }}\right)$ [Table S1]. The ratio of the two measured values was used to calculate the catalytic efficiency $\left(k_{\text {cat }} / K_{\mathrm{M}}\right)$ for all protease variants. Wild-type protease had a $K_{\mathrm{M}}$ of $71.4 \mu \mathrm{M}$ and $k_{\text {cat }}$ of $1282.7 \mathrm{~s}^{-1}$, yielding a catalytic efficiency of $17.1 \mu \mathrm{M}^{-1}$ $\mathrm{s}^{-1}$. 1Mut, 2Mut, and 4Mut variants had WT-like $K_{\mathrm{M}}$ values. However, $k_{\text {cat }}$ of these variants were 2-7-fold lower than WT protease, resulting in decreased catalytic efficiency. The $2 \mathrm{Mut}$ variant had a 5-fold decrease in $k_{\text {cat }} / K_{\mathrm{M}}$ compared to WT ( $k_{\text {cat }} /$ $\left.K_{\mathrm{M}}=3.4 \pm 0.2 \mu \mathrm{M}^{-1} \mathrm{~s}^{-1}\right)$.

Relative to WT protease, $K_{\mathrm{M}}$ values of the highly mutated 8Mut, 9Mut-A71V, 9Mut-L76V, and 10Mut variants increased 1.4-2.4-fold. The $k_{\text {cat }}$ value of the 8Mut and 10Mut decreased 2-3-fold, and the $k_{\text {cat }} / K_{\mathrm{M}} 3-5$-fold relative to WT. The most striking loss was in the turnover rates of the 9Mut variants, with 2 orders of magnitude slowing in the cleavage rate $\left(k_{\text {cat }}=\right.$ $16.9 \pm 0.3$ and $17.7 \pm 0.3$, respectively, for 9Mut-A71V and 9 Mut-L76V). Combined with the increase in $K_{\mathrm{M}}$, these variants were extremely inefficient with $k_{\text {cat }} / K_{\mathrm{M}}$ values 171 fold lower relative to $\mathrm{WT}$, representing a severe enzymatic penalty for these intermediate variants that were undetectable in viral passaging. Strikingly, co-occurrence of the A71V and L76V mutations in the 10Mut variant restored catalytic efficiency $\left(k_{\mathrm{cat}} / K_{\mathrm{M}}=5.3 \pm 0.1 \mu \mathrm{M}^{-1} \mathrm{~s}^{-1}\right)$. The $11 \mathrm{Mut}$ exhibited restored WT-like $K_{\mathrm{M}}$ but was 13 -fold less catalytically efficient compared to WT protease $\left(k_{\text {cat }} / K_{\mathrm{M}}=1.3 \pm 0.4 \mu \mathrm{M}^{-1}\right.$ $\left.\mathrm{s}^{-1}\right)$.

This enzymatic assay uses a single cleavage site for a relatively short amount of time. To examine how the highly mutated variants process multiple cleavage sites in full length Gag polyprotein ( $\mathrm{p} 55$ ) over a longer time scale, a gel cleavage assay was utilized ${ }^{35,36}$ [Figure S1]. Wild-type protease was extremely efficient, cleaving $100 \%$ of the p55 polyprotein after $90 \mathrm{~min}$. All the mutants were able to cleave the initial p2-NC cleavage site at varying rates. The intermediate 9 Mut variants were much slower but were still able to process the multiple cleavage sites in the polyprotein. Overall, all protease variants were able to process substrates, with the highly mutated and abundant 10Mut and 11Mut variants having restored catalytic activity.

Accumulation of Mutations Progressively Increases DRV Resistance. The enzyme inhibition constant, $K_{\mathrm{i}}$, of DRV was measured against WT and the selected protease variants using an established assay with an optimized fast-cleaving substrate. ${ }^{37}$ Wild-type protease is highly susceptible to DRV with a $K_{\mathrm{i}}$ in single digit picomolar range below the assay limit of reliable detection $\left(K_{\mathrm{i}}<0.005 \mathrm{nM}\right)$, as we previously reported. ${ }^{38}$ Against 1Mut, 2Mut, and 4Mut, DRV maintained picomolar inhibition, but with $K_{\mathrm{i}}$ increasing progressively 680 -fold relative to $\mathrm{WT}$ [Figure 1C]. The 8 Mut variant was significantly more resistant to DRV, with a 2560 -fold increase in resistance compared to WT $\left(K_{\mathrm{i}}=12.8 \mathrm{nM}\right)$. Addition of A71V mutation (9Mut-A71V) resulted in a $\sim 2$-fold further increase in resistance $\left(K_{\mathrm{i}}=23.2 \mathrm{nM}\right)$. In contrast, addition of L76V (9Mut-L76V) caused an order of magnitude increase in DRV resistance $\left(K_{\mathrm{i}}=172.7 \mathrm{nM}\right)$, indicating that the distal L76V mutation contributes a significant level of resistance. The 10 Mut variant harboring both distal mutations exhibited 9MutL76V-like inhibition $\left(K_{\mathrm{i}}=156.4 \mathrm{nM}\right)$. Addition of I54L in 11 Mut resulted in dramatic DRV resistance, with a 152000fold decrease in inhibition compared to WT $\left(K_{\mathrm{i}}=759.2 \mathrm{nM}\right)$. Active site mutations alone do not account for the high levels of resistance seen in the highly mutated variants, as only with the addition of distal mutations the protease was able to exhibit nanomolar-level inhibition. The relationship between DRV inhibition and catalytic efficiency is shown in Figure S2.

Double Mutant Cycle Reveals the Interdependency of A71V and L76V. Only the 8Mut and 10Mut variants were observed in viral passaging; thus the addition of $\mathrm{A} 71 \mathrm{~V}$ and L76V mutations individually were examined in a double mutant cycle analysis [Figure 1D] to elucidate the interdependency of these two distal mutations, which cause 12 -fold increase in resistance. The change in free energy of inhibitor binding (estimated from $\left.\Delta G=R T \ln \left[K_{\mathrm{i}}\right]\right)^{39}$ between the reference variant 8 Mut and 10 Mut $\left(\Delta \Delta G_{10 \mathrm{Mut}}\right)$ was calculated. This was then compared to the sum of individual changes for $9 \mathrm{Mut}-\mathrm{A} 71 \mathrm{~V}$ and $9 \mathrm{Mut}-\mathrm{L} 76 \mathrm{~V}$ relative to $8 \mathrm{Mut}$ 
$\left(\Delta \Delta G_{9 \text { Mut-A71V }}+\Delta \Delta G_{9 \text { Mut-L76V }}\right)$. When the two mutations are independent, the two values are equal $\left(\Delta \Delta G_{10 \mathrm{Mut}}=\right.$ $\left.\Delta \Delta G_{9 \text { Mut-A71V }}+\Delta \Delta G_{9 \text { Mut-L76V }}\right)$. The sum of free energy changes for the 9Mut intermediates was more than that for 10Mut [Figure 1D]. $\Delta \Delta G_{9 \text { Mut-A71v }}$ was $0.35 \pm 0.07 \mathrm{kcal} / \mathrm{mol}$ and $\Delta \Delta G_{9 \text { Mut-L76V }}$ was $1.55 \pm 0.10 \mathrm{kcal} / \mathrm{mol}$, summing to 1.90 $\pm 0.12 \mathrm{kcal} / \mathrm{mol}$; however, $\Delta \Delta G_{10 \mathrm{Mut}}$ was $1.45 \pm 0.05 \mathrm{kcal} /$ mol. Thus, the double mutant cycle analysis shows that A71V and $\mathrm{L} 76 \mathrm{~V}$ are interdependent ${ }^{39}$ in conferring DRV resistance.

Crystal Structures Show Resistance Mutations Reduce vdW Contacts with DRV. To elucidate the changes in inhibitor binding and molecular mechanisms of resistance, we determined cocrystal structures of DRV bound to the wild-type HIV-1 protease of the NL4-3 strain, as well as all eight mutated variants at high resolution (1.9-2.2 $\AA$ ). Structure of DRV bound to WT protease and the I84V variant were previously determined $^{22}$ and the $2 \mathrm{Mut}$ crystallized in the same space group $\left(P 2_{1} 2_{1} 2_{1}\right)$, which contained one homodimer in the asymmetric unit and one inhibitor bound in a single orientation, allowing direct comparison. The variants containing four or more mutations had a different preferred crystallographic lattice and were solved in the $P 6_{1}$ space group. For reliable and direct comparison with these variants, WT protease structure was also determined in the hexagonal space group. These structures were also solved containing one homodimer in the asymmetric unit with one inhibitor bound in a single orientation [Table S2]. Structures of protease variants were overall similar to that of WT protease, with $0.3-0.4 \AA$ overall RMSD of $\mathrm{C}_{\alpha}$ atoms [Figure S3]. Following established convention, throughout this analysis the $\mathrm{B}$ chain that contacts the aniline moiety of DRV will be denoted the prime (') chain, while the A chain will remain nonprime. The crystal structures determined provided insights into changes in the overall structure and protein-inhibitor interactions due to the accumulating resistance mutations.

Resistance mutations selected under DRV selective pressure are expected to directly or indirectly perturb inhibitor binding and result in reduced potency. To elucidate alterations in inhibitor packing at the active site, we calculated vdW contacts of DRV with the protease residues from the crystal structures [Table S3]. Compared to WT protease, the common multidrug resistance mutation $184 \mathrm{~V}$ resulted in a loss of 1.2 and $1.8 \mathrm{kcal} / \mathrm{mol}$ in $\mathrm{vdW}$ contacts of residue 84 and $84^{\prime}$, respectively [Figure S4A, Table S3]. Similarly, all remaining variants, which also contain this mutation, had $1-2.5 \mathrm{kcal} / \mathrm{mol}$ losses in vdW contacts at these two residues. In the presence of the single I84V mutation, the location of the other active site mutation, V82, had a compensatory gain in vdW contacts in chain $\mathrm{B}$, which was progressively alleviated as the mutations were added. Overall, V82F exhibits a minor gain in vdW contacts $(0.2 \mathrm{kcal} / \mathrm{mol}$ on average $)$, but the larger nonpolar side chain significantly shifts Arg8' away from DRV resulting in $0.6-1.1 \mathrm{kcal} / \mathrm{mol}$ loss in $\mathrm{vdW}$ contacts. Addition of flap mutations K45I and M46I caused reduced vdW contacts of I50 in 4Mut variant and a net loss in total vdW contacts, which correlates with reduced potency [Figure S4B, Table S3]. The highly resistant 8 Mut variant structure continued the trend, exhibiting a net reduction in vdW contacts. Notably, although the V32I mutation results in a larger amino acid at the edge of the active site, vdW contacts with DRV were reduced $0.4-0.6$ and $0.5-1.0 \mathrm{kcal} / \mathrm{mol}$ due to the orientation of the additional methyl group away from the inhibitor [Figure S4A, Table S3]. The combination of I13V, L33F, and V32I exacerbated losses of $\mathrm{vdW}$ contacts at residues $\mathrm{I} 84 \mathrm{~V}$ and $\mathrm{I} 84 \mathrm{~V}^{\prime}$ by 0.2 and 0.7 $\mathrm{kcal} / \mathrm{mol}$, respectively. Each mutation, both proximal and distal, either directly or indirectly reduced vdW contacts with the inhibitor, thereby destabilizing inhibitor binding.

Although the 9Mut variants were not observed in viral passaging, determining the structures of these complexes highlighted the interdependent structural changes of the mutations at residue 71 and 76 connecting the $8 \mathrm{Mut}$ and $10 \mathrm{Mut}$ variants. The A71V mutation, located on the distal "70's loop", is a well-known compensatory mutation that restores catalytic activity of protease variants containing I50V mutation. The differences in internal $\mathrm{C}_{\alpha}$ distances [Figure S5], also known as distance difference matrices, were calculated to quantify the structural alterations. Comparing the 8 Mut and 9Mut-A71V structures, the A71V mutation pushes the flexible "70s loop" outward up to 0.61 and $0.63 \AA$ for residues 67 and $68^{\prime}$, respectively. This outward shift can perturb the $\beta$ strand (residues 69-76) that directly interacts with the neighboring $\beta$ strand extending up to the flaps (residues 53-65). Although there are very few changes in the internal $\mathrm{C}_{\alpha}$ distances of $8 \mathrm{Mut}$ and 9 Mut-L76V, when we compare 8 Mut and 10 Mut the changes in distances reflect an additivity of the differences between the 8 Mut versus the two 9Mut structures [Figure S5].

Comparing the complexes of 10Mut and 11Mut helps explain the 5-fold reduction in potency. The longer side chain resulting from the I54L mutation pushed against Pro79 creating a mean difference of $0.36 \AA$ at residues 54 and $54^{\prime}$ and $0.20 \AA$ at residues 79 and $79^{\prime}$ [Figure S5]. This widening at the active site may destabilize flap closing, and most notably resulted in reduced vdW contacts at residues 49 and 50 of 0.9 and $0.8 \mathrm{kcal} / \mathrm{mol}$, respectively. 11Mut-DRV cocrystal structure had the lowest total intermolecular vdW contacts, correlating with the highest DRV resistance.

Resistance Mutations Perturb Packing in the Hydrophobic Core. Many of the mutations observed were changes from one hydrophobic side chain to another, located in the hydrophobic core of the protein, that considerably change the packing between hydrophobic side chains. K45I results in a side chain rotamer that increases hydrophobic packing with residues I47, V56, and L76 at the base of the flaps [Figure S4B]. While I84V may only bridge the gap between V82F and V32I [Figure S4A], V32I is involved in both the L76V cluster (also including K45I, I47, and V56) [Figure S4C] and the I54L cluster (also including I47, I50, and V56) [Figure S4D]. Mutations that perturb the packing of these clusters may also affect "hydrophobic sliding" and thus conformational dynamics of the protease. ${ }^{40,41}$

Resistance Mutations Increase Protease Fluctuations. Despite 5 orders of magnitude increase in the inhibition constant $\left(K_{\mathrm{i}}\right)$ of the $11 \mathrm{Mut}$ variant relative to WT protease, DRV displayed only minor conformational differences in the cocrystal structures, and $\mathrm{MD}$ simulations were performed to better interrogate these structures. Starting from the cocrystal structures of the DRV complexes with WT protease and the resistant variants, three replicates of fully hydrated $100 \mathrm{~ns} \mathrm{MD}$ simulations were performed for each complex. All simulations reached convergence [Figure S6]. The protein backbone dynamics were compared via root-mean-square fluctuations (RMSF) of $\mathrm{C}_{\alpha}$ atoms [Figure S7]. In WT-DRV complex, the entire backbone was stable, including the flaps (50s region) that close upon the bound inhibitor. In I84V variant, the flaps wre slightly more mobile but the active site remained stable. Relative to $\mathrm{I} 84 \mathrm{~V}$, the $2 \mathrm{Mut}(\mathrm{I} 84 \mathrm{~V} / \mathrm{V} 82 \mathrm{~F})$ protease had 

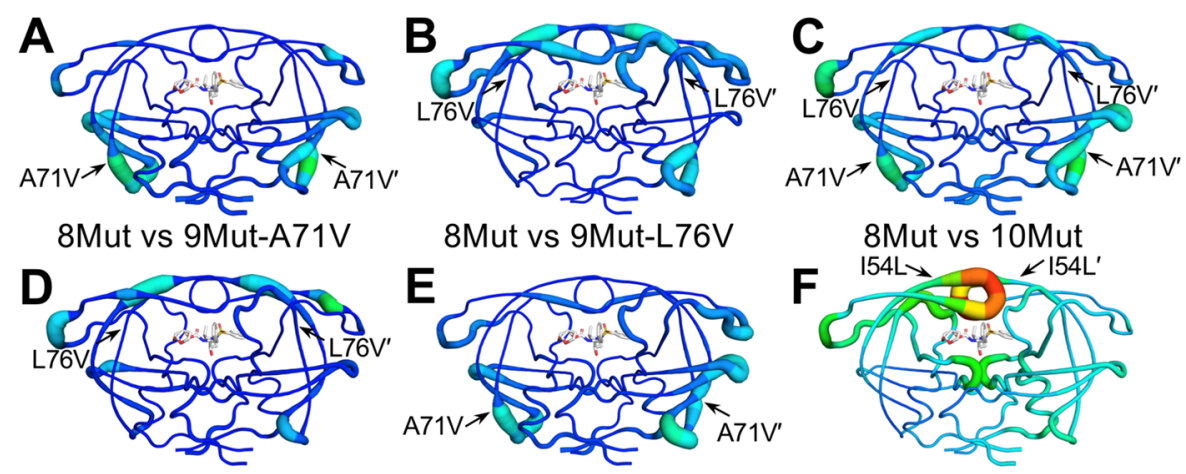

9Mut-A71V vs 10Mut 10Mut vs 9Mut-L76V

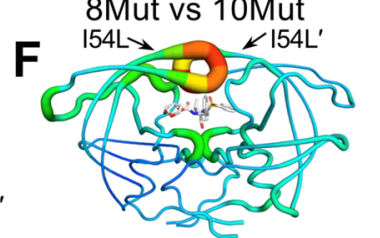

10Mut vs 11Mut

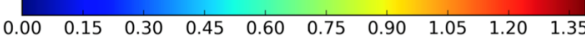

C-Alpha Distance Difference $(\AA)$

Figure 2. Mean internal $\mathrm{C}_{\alpha}$ distance differences between (A) 8Mut and 9Mut-A71V, (B) 8Mut and 9Mut-L76V, (C) 8Mut and 10Mut, (D) 9MutA71V and 10Mut, (E) 10Mut and 9Mut-L76V, and (F) 10Mut and 11Mut protease variants from MD simulations plotted onto protease structure. In all panels, DRV is shown as sticks in the active site. Putty thickness and color indicate distance difference where hot colors indicate large changes.

reduced flap fluctuations [Figure S8]. The addition of flap mutations $\mathrm{K} 45 \mathrm{I}$ and M46I in the 4Mut resulted in higher fluctuations at chain A flap and residues 79-82. Chain B flap and residues $79^{\prime}-82^{\prime}$ had WT-like fluctuations, remaining stable throughout the MD simulations. Overall, the backbone flexibility of the highly mutated 8Mut, 9Mut-A71V, 9MutL76V, and 10Mut variants were similar to WT complex, except increased fluctuations at residue $65^{\prime}$ in 10 Mut. 11 Mut variant, bearing flap mutation I54L, displayed extremely large fluctuations at both flaps (residues $42-55$ and $45^{\prime}-52^{\prime}$ ), increasing more than 0.6 and $0.5 \AA$ in chain $\mathrm{A}$ and $\mathrm{B}$, respectively, relative to $\mathrm{WT}$ protease. In addition, $11 \mathrm{Mut}$ protease had increased fluctuations at residues $79-82$ and $78^{\prime}-80^{\prime}$ as well as residues $26-28$ in the active site. Thus, the addition of I54L mutation resulted in considerable increase in protease backbone fluctuations at the active site and flaps.

To compare the dynamic ensemble of DRV-bound variants, mean internal $\mathrm{C}_{\alpha}$ distances from $\mathrm{MD}$ simulations were compared, similar to the comparison of crystal structures [Figure 2]. In this analysis, the internal distances between all $\mathrm{C}_{\alpha}$ carbons were measured for every frame of the trajectory and an average was taken. Comparing $8 \mathrm{Mut}$ to $9 \mathrm{Mut}-\mathrm{A} 71 \mathrm{~V}$, changes were mostly localized to the $70 \mathrm{~s} \beta$-sheets and residues 15-20 in both chains, with small changes at the flap elbows [Figure 2A]. Comparing 8Mut to 9Mut-L76V, changes were located in both flaps and the flap elbows. The 80s loop (residues 78 to 82 ) also had slight changes. In addition, the B chain $70 \mathrm{~s} \beta$-sheet was affected while the $\mathrm{A}$ chain $\beta$-sheet showed very little change [Figure $2 \mathrm{~B}$ ]. As was observed with the crystal structures, alterations in the two 9-Mut variants were additive when 10Mut was compared to 8 Mut. The exception was that changes at residues $78-82$ were attenuated when $\mathrm{A} 71 \mathrm{~V}$ and $\mathrm{L} 76 \mathrm{~V}$ were simultaneously present [Figure $2 \mathrm{C}]$. To complete the cycle, the two 9 Muts were compared with 10Mut. Similar to the effects noted earlier, A71V induced changes at the 70 s $\beta$-sheets and residues $15-20$, while L76V distally affected the flaps and flap elbows [Figure 2D,E]. These alterations in the dynamic ensemble were consistent with the comparison of crystal structures [Figure S5]. However, alterations in the $11 \mathrm{Mut}$ variant were accentuated in the conformational dynamics in the MD simulations, with large structural changes at both flaps and the active site [Figure 2F].
Compared to other variants, the flaps of 11 Mut were more open as indicated by the increasing distance between the $\mathrm{C}_{\alpha}$ atoms of I50-V84' and I50'-V84 [Figure S9]. Thus, I54V mutation in 11 Mut resulted in destabilization and increased fluctuations of the protease, particularly in the flaps.

Inhibitor Fluctuations Increase and Interactions Are Destabilized Due to Resistance Mutations. In the cocrystal structures, conformation of DRV was highly similar, with the most notable alterations occurring at the $\mathrm{P} 1^{\prime}$ moiety [Figure S10]. To investigate how the dynamics of DRV may vary, the RMSF of every DRV heavy atom from the MD simulations was calculated and grouped according to the four DRV moieties [Figure 3]. In complex with WT protease DRV was stable, with RMSF below $0.75 \AA$ A except for the P1' moiety. In $1 \mathrm{Mut}, 2 \mathrm{Mut}$, and $4 \mathrm{Mut}$ variants, fluctuations of DRV moieties were similar to those in WT complex, with few minor exceptions at the $\mathrm{P} 1^{\prime}$ and $\mathrm{P} 1$ moieties (data not shown). When bound to all the highly mutated variants, DRV had increased flexibility, especially at the P1 and P2 moieties [Figure 3]. In 10Mut complex, the P2 bis-THF moiety had high RMSF compared to WT and all other variants. This moiety makes several hydrogen bonds to protease active site residues and contributes significantly to the high potency of DRV. In complex with the 11 Mut variant, the entirety of DRV experienced significant flexibility with the P1 moiety showing the greatest RMSF. Overall, resistance mutations in the protease variants resulted in increased flexibility and higher fluctuations of DRV, suggesting that inhibitor binding and intermolecular interactions are destabilized.

Underlying the high potency of DRV against WT HIV-1 protease is an extensive hydrogen bonding network and strong vdW interactions with active site residues. In highly mutated variants, these interactions may be perturbed, as suggested by increased DRV fluctuations. The vdW packing around DRV in complex with $8 \mathrm{Mut}$, 9Mut-A71V, 9Mut-L76V, and 10Mut decreased slightly around the P2 bis-THF moiety compared to WT [Figure 3B]. In 11Mut variant, packing around DRV was significantly reduced at the $\mathrm{P} 1$ phenyl, $\mathrm{P} 1^{\prime}$ isobutyl, and $\mathrm{P} 2^{\prime}$ aniline moieties. In the cocrystal structure bound to WT protease, DRV makes a number of hydrogen bonds with the protease backbone and a water-mediated hydrogen-bonding network with the flaps [Figure 4A]. All these hydrogen bonds 


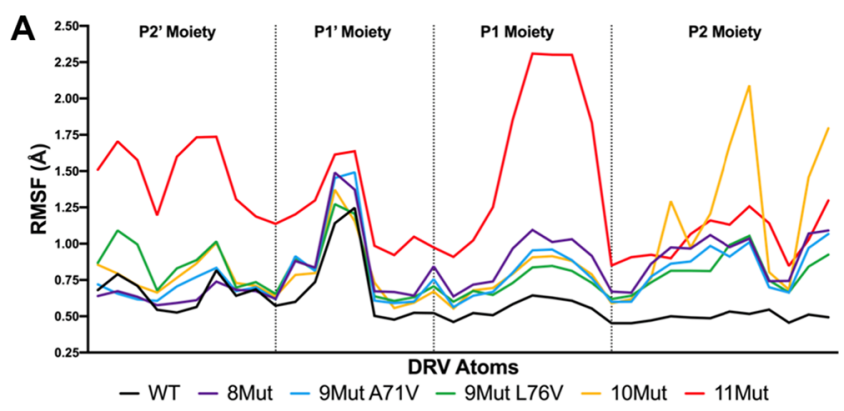

B
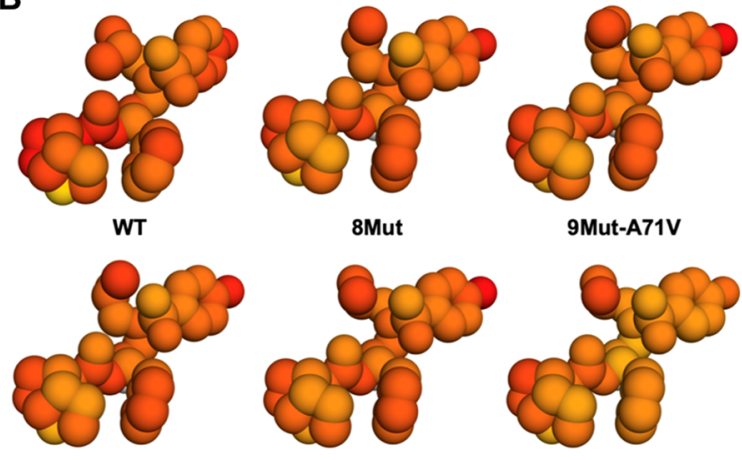

10Mut

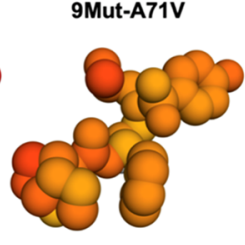

11Mut

Figure 3. (A) Root-mean-square fluctuation (RMSF) of DRV atoms grouped by moiety monitored during MD simulations bound to WT and resistant HIV-1 protease variants (color scheme as in Figure 1). (B) Packing around DRV in complex with WT protease and resistant variants. Total per atom protease-DRV vdW contact energies mapped onto the respective DRV crystal structure, with red indicating more contacts.

were stable and highly conserved throughout the MD simulations [Figure 4B]. In the highly mutated variants, frequency of hydrogen bonds during the MD simulations decreased relative to WT complex, with a severe loss in the hydrogen bond between $\mathrm{D} 25^{\prime}$ and the central hydroxyl of DRV. In 8Mut and 9Mut-A71V variants, DRV hydrogen bonds with $\mathrm{P} 2$ bis-THF and flaps destabilized $8-17 \%$, and frequency of the hydrogen bond between $\mathrm{P} 2$ ' aniline and backbone nitrogen of $\mathrm{D} 30^{\prime}$ decreased $18 \%$. In $9 \mathrm{Mut}-\mathrm{L} 76 \mathrm{~V}$ and $10 \mathrm{Mut}$ variants, there was a further decrease in the frequency of these hydrogen bonds. In 11Mut variant, water-mediated hydrogen bonding network with the flaps was severely impacted, where two hydrogen bonds were completely lost and the other two maintained only $25 \%$ during the MD simulations. The hydrogen bond between D25 and the central hydroxyl was reduced to $62 \%$ where it was previously maintained at $83-99 \%$ for WT and all other resistant variants. Additionally, the P2' aniline hydrogen bond with $\mathrm{D} 30^{\prime}$ was significantly reduced to only $9 \%$, an $85 \%$ decrease compared to WT. Thus, the impact of distal mutations in these highly mutated and resistant variants propagated to the active site to destabilize inhibitor $\mathrm{vdW}$ and hydrogen bonding interactions with the protease.

Protease-DRV Cross-Correlations of Fluctuations Are Lost with Increasing Resistance. Cross-correlation of collective motions between a ligand and target protein can distinguish tight and weak binders, as we previously reported. ${ }^{28,30,42}$ For HIV-1 protease, tight binding inhibitors have greater correlation of fluctuations with proximal protease residues, namely, the active site and flaps. Contrary to tight binders, weak binding inhibitors lose correlations with these residues corresponding to disrupted intermolecular interactions. ${ }^{30}$ Cross-correlations between the fluctuations of DRV and protease residues were calculated from the MD simulation trajectories. As expected, DRV fluctuations were highly correlated with catalytic residues especially at chain A, flap tips $\left(49-51 / 49^{\prime}-51^{\prime}\right)$, and residues $26-31$ adjacent to the catalytic D25 [Figure 5A]. DRV also had moderate correlations with residues $81-87$, while the flap elbows and 70 s $\beta$-sheets were anticorrelated. In $8 \mathrm{Mut}$, DRV maintained correlation with residues $49^{\prime}-50^{\prime}$ but decoupled from chain A flap tip and the active site residues [Figure S11]. While 9MutA71V restored the lost correlations of DRV with the catalytic residues, the flap tips became further decoupled. In 9MutL76V and 10Mut, DRV fluctuations were further decoupled from the flap tips, but in both variants DRV somewhat maintained correlation with catalytic residues [Figure 5]. In 11 Mut variant, positive correlations with DRV were almost completely lost, and the inhibitor established anticorrelations with various regions of the protein (chain B flap, S2' subsite, chain B 70s $\beta$-sheet and 80s loop). Disruptions in the correlations between DRV and 11Mut fluctuations are consistent with increased resistance and weaker intermolecular interactions.

Distal Mutations Impact the Dynamic Ensemble to Confer Resistance. The existing paradigm reasons that distal mutations play a compensatory role in HIV-1 protease, acting to restore catalytic efficiency lost by the accumulation of mutations. However, while certain distal mutations indeed play a compensatory role, they also confer resistance as demonstrated above. We reported previously that distal mutations may propagate their effects to the active site via altering the conformational dynamics of the inhibitor-protease
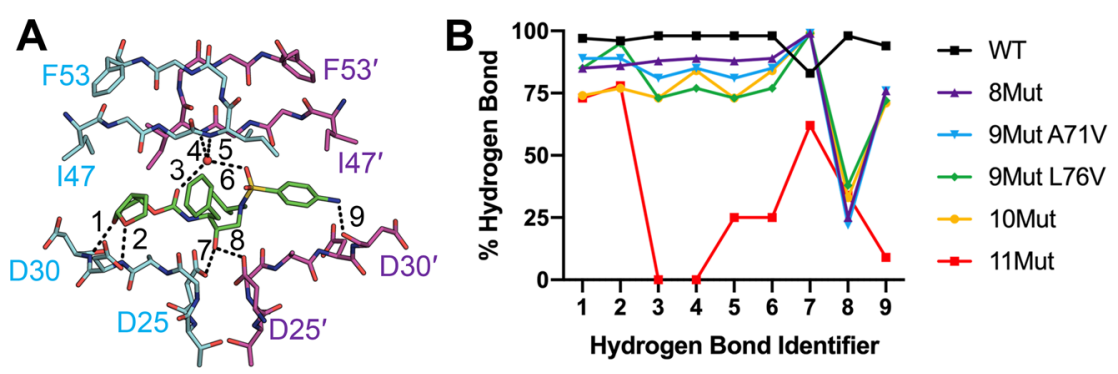

Figure 4. Resistant HIV-1 protease variants lose hydrogen bonding with inhibitor DRV. (A) Hydrogen bonds with DRV (green sticks) indicated as black dashed lines on the WT protease crystal structure. The "flap water" molecule is depicted as a red sphere. Numbers correspond to hydrogen bonds shown in panel B. (B) Hydrogen bonding frequencies from MD simulations. The water-mediated hydrogen-bonding network between the protease flaps and DRV is severely disrupted in 11Mut. 

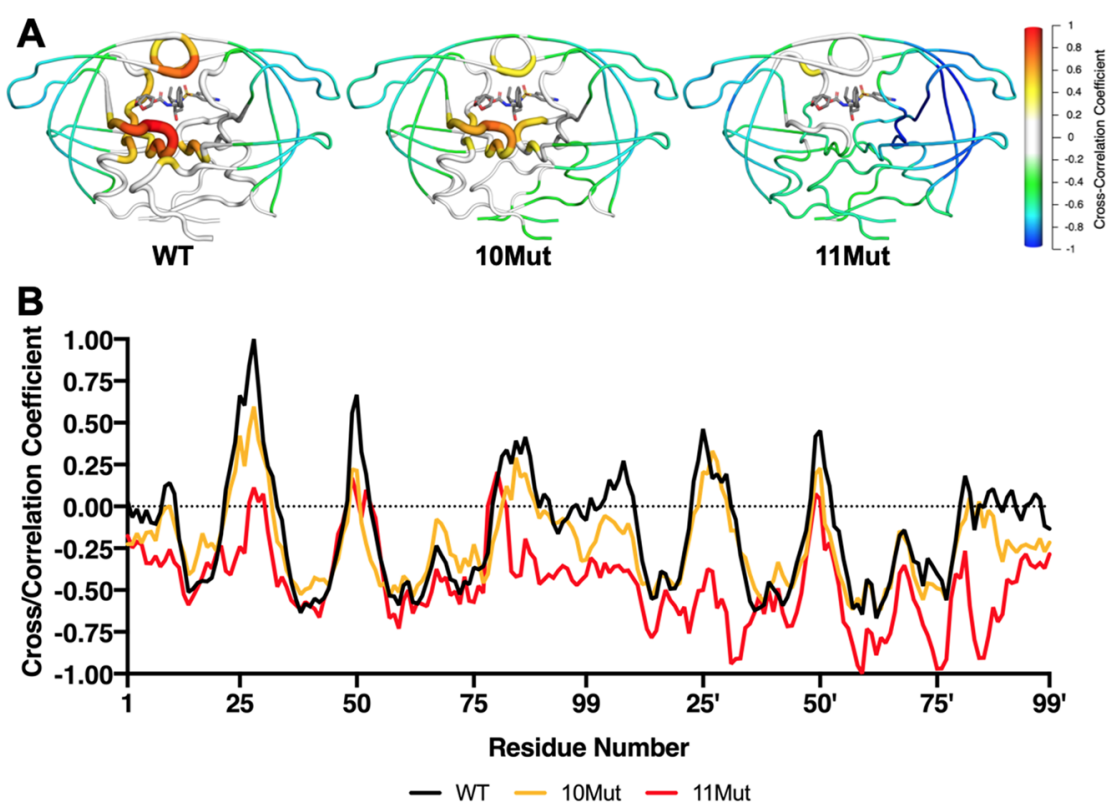

Figure 5. Cross-correlation of fluctuations between HIV-1 protease and DRV from MD simulations. (A) Mean cross-correlation coefficient for each residue mapped onto protease structure, shown as cartoon putty for WT, 10Mut, and 11Mut variants. (B) Cross-correlations for WT, 10Mut, and 11 Mut plotted. As resistance mutations accumulate, positive correlations decrease while negative correlations increase.

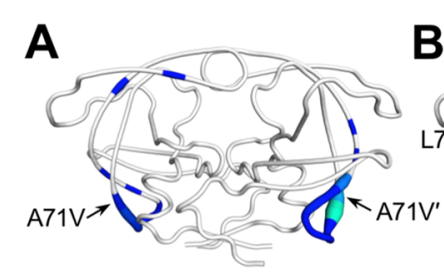

8Mut vs 9Mut-A71V

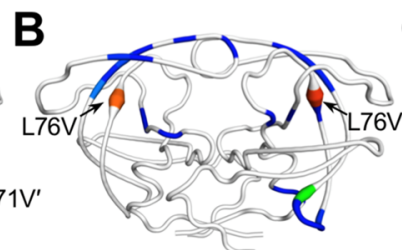

8Mut vs 9Mut-L76V

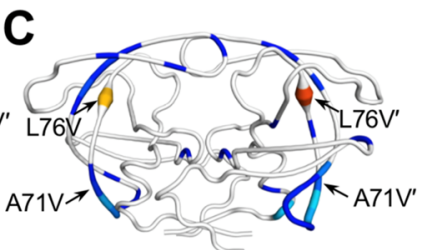

8 Mut vs 10 Mut

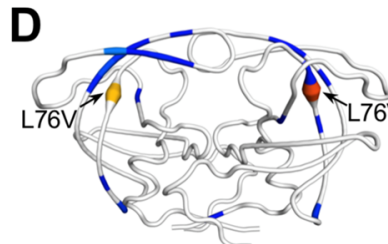

9Mut-A71V vs 10Mut
$\mathbf{E}$

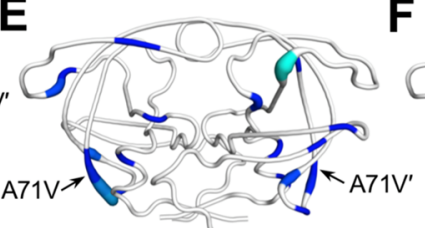

9Mut-L76V vs 10Mut

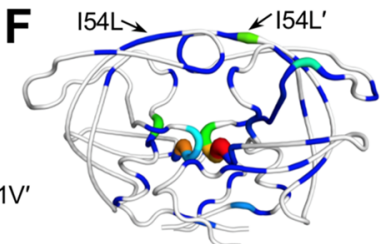

10Mut vs 11Mut

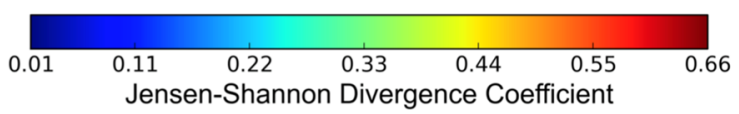

Figure 6. Comparison of protease dynamics via Jensen-Shannon divergence of dihedral angles between (A) 8Mut and 9Mut-A71V, (B) 8Mut and 9Mut-L76V, (C) 8Mut and 10Mut, (D) 9Mut-A71V and 10Mut, (E) 9Mut-L76V and 10Mut, and (F) 10Mut and 11Mut from MD simulations, mapped onto protease structure. Tube thickness and warmer colors indicate larger perturbation of the dynamic ensemble. Residues in white had no significant difference between the two variants. Arrows denote location of mutation(s) different between the variants compared.

complex, via hydrophobic sliding ${ }^{40,41}$ and the internal hydrogen bonding network. ${ }^{29}$ Here we used Jensen-Shannon divergence (JSD) to analyze perturbations in the dynamic ensemble in response to mutations, where protein backbone and side chain dihedral angles were compared between variants during MD simulations. We focused on the impact of 3 additional nonactive site mutations, from $8 \mathrm{Mut}$ to $11 \mathrm{Mut}$, which results in almost 60 -fold increase in resistance.

Consistent with the $\mathrm{C}_{\alpha}$ distance difference analysis, A71V caused only local perturbations in the dynamic ensemble in the $70 \mathrm{~s} \beta$-sheets [Figure 6A]. L76V also perturbed the dynamics of the 70s loop (chain B) but also had distal effects on the dihedrals of residues in both flaps [Figure 6B]. In combination, the effects of $\mathrm{A} 71 \mathrm{~V}$ and $\mathrm{L} 76 \mathrm{~V}$ were mostly additive, but together they also perturbed the side chain dihedrals of catalytic D25/25' residues ( $\phi$ of D25 and $\chi^{2}$ of D25') [Figure $6 \mathrm{C}]$. However, the alterations in D25/D25' were subtle and did not significantly change the hydrogen bonds with DRV [Figure 4]. Comparison of 9Mut variants with 10Mut confirmed the earlier observation that addition of L76V mostly impacted the flaps [Figure 6D], while A71V had local effects on the proximal $70 \mathrm{~s} \beta$-sheets [Figure $6 \mathrm{E}$ ]. Lastly, addition of I54L in 11 Mut caused considerable changes to the dynamic ensemble compared to 10Mut, both proximal and distal. Most noticeably, side chain dihedrals of catalytic residues were impacted $\left(\psi\right.$ and $\chi^{2}$ of D25 and $\chi^{2}$ of D25'), which is 
consistent with decreasing hydrogen bonding between the catalytic residues and the central hydroxyl of DRV [Figure 4]. In addition to changes at the active site, the dynamic ensemble of the flaps and 80s loop of chain B were affected. These results indicate that distal resistance mutations propagate their effects throughout the protein structure, which can be captured as alterations to the dynamic ensemble of protein dihedral angles.

\section{DISCUSSION}

Drug resistance occurs when the target evolves and gains mutations to thwart inhibition while still maintaining biological function. We investigated a highly evolved and mutated variant of HIV-1 protease that accumulated 11 mutations to confer high-level resistance to DRV. We systematically dissected the mutations in this variant by generating 8 different subset variants, including separating active site mutations from distal mutations and leveraging a double mutant cycle analysis [Figure 1A]. Integrating activity and inhibition assays, highresolution crystal structures, and molecular dynamics simulations, we determined how active site and distal mutations contribute to drug resistance. Our results show that resistance progressively increased as the mutations were added, while catalytic activity was maintained. Mutations were selected both inside and outside the active site where the inhibitor binds. We found that the distal mutations play a pivotal role in conferring drug resistance, as proximal mutations alone cannot explain the observed near micromolar resistance. Double mutant cycle analysis revealed that two such distal mutations are interdependent, with $\mathrm{A} 71 \mathrm{~V}$ restoring catalytic activity and L76V decreasing inhibition. Our findings challenge the convention that distal mutations are merely compensatory and have secondary or minimal contribution to resistance. On the contrary, we demonstrated that distal mutations act interdependently through complex molecular mechanisms to confer high levels of resistance.

In addition to avoiding inhibition, the mutated protease needs to continue processing viral substrates to allow viral replication. The highly mutated variants (8Mut, 10Mut, $11 \mathrm{Mut}$ ) detected from viral passaging had somewhat compromised enzymatic activity relative to WT protease, although still allowed for robust viral replication. Unlike the detectable variants, both 9 Mut-A71V and 9 Mut-L76V variants had severely compromised catalytic activity. The protease must evolve through one of the 9Mut intermediates en route from 8 Mut to 10 Mut variant, but the virus cannot efficiently replicate to populate detectable levels. $\mathrm{A} 71 \mathrm{~V}$ is a well-known compensatory mutation that is often observed together with I50V and was reported to restore catalytic activity lost due to this primary mutation. ${ }^{43,44}$ The 8 Mut variant does not contain I50V, and when added to the $8 \mathrm{Mut}$ variant A71V actually obliterated catalytic activity (9Mut-A71V). In the presence of the $\mathrm{L} 76 \mathrm{~V}$ mutation though the catalytic activity was restored (10Mut). Thus, the effect of A71V on catalytic activity is context dependent and may be beneficial or detrimental depending on the background mutation(s).

While resistance due to primary active site mutations are rather straightforward to explain especially using the substrate envelope, how combinations of mutations including distal mutations act interdependently to confer resistance requires further investigation. The crystal structures showed consistent losses in protein-inhibitor vdW contacts, differences in internal distances, and subtle alterations in the binding conformation of DRV. However, given the high levels of resistance and orders of magnitude change in inhibition constants, we expected further and amplified impact on the conformational dynamics of the protease-inhibitor complex. MD simulations highlighted the extent of unfavorable protease-DRV interactions with increasing mutation accumulation. Highly mutated variants resulted in increased protein and inhibitor fluctuations and, most importantly, reduced cross-correlated motions. We previously found that the loss in correlated inhibitor-target fluctuations correlated with reduced inhibitor potency for HCV NS3/4A protease $^{30}$ and analogous inhibitors binding to WT HIV-1 protease. ${ }^{28}$ We find here that the same loss exists for highly mutated and resistant variants of HIV-1 protease. In addition, the hydrogen bonds and vdW interactions that contribute to DRV's picomolar potency in WT HIV-1 protease were greatly diminished. Alterations in the dynamics of the protease-inhibitor complex are crucial in the molecular mechanisms of resistance, and thus the molecular dynamics need to be investigated in addition to static crystal structures to elucidate how the effect of distal mutations can be propagated to cause alterations in inhibitor binding.

Here we develop an alternative approach to previously explored methods to elucidate how distal mutations propagate dynamic changes throughout the protease. Expanding upon hydrophobic sliding ${ }^{40,41}$ and internal hydrogen bonding networks, ${ }^{29}$ quantitative comparison of protein dihedral angles can be used to examine dynamic structural changes. As dihedral angles from MD simulations comprise highly similar probability distributions, a rigorous statistical analysis involving Jensen-Shannon divergence implementation is needed to determine distinct changes. This approach previously quantified differences in long-range dynamics of proteins with and without a ligand bound. ${ }^{45}$ Dihedral angle comparison illustrated the propagation of dynamic effects due to distal mutations throughout the protein, including impact of A71V/ L76V on the catalytic residues. In the most mutated and most resistant variant $11 \mathrm{Mut}$, addition of the I54L mutation conferred many additional changes to the active site and flaps while also propagating changes throughout the protease structure. This analysis demonstrated how mutations cause dynamic changes that propagate to distal regions of the protein, and thus how considering local changes to the static structure is not sufficient to elucidate molecular mechanisms of drug resistance.

Our work provides evidence that distal mutations do indeed contribute to drug resistance through complex dynamic processes, significantly altering protease dynamics and perturbing inhibitor binding. In other HIV-1 protease variants and, perhaps more significantly, other drug targets where distal mutations are observed in clinically relevant resistant variants, the possibility that distal mutations directly confer resistance cannot be ignored. This also has the implication that drug design needs to consider both structural and dynamic changes to the protein-inhibitor complex to be able to effectively target these variants. The increasing threat of drug resistance in many clinically relevant systems highlights the need to characterize resistance mutations, including distal mutations and combinations of mutations that interdependently confer resistance through complex dynamic resistance mechanisms.

\section{METHODS}

Protein Preparation. Protease Gene Construction. Protease gene construction was carried out as previously described. ${ }^{30,46}$ In 
short, the WT and 10Mut protease variant genes were purchased on a pET11a plasmid with codon optimization for protein expression in $E$. coli (Genewiz). The remaining variants were constructed using QuikChange site-directed mutagenesis (Genewiz). A Q7K mutation was included to prevent autoproteolysis. ${ }^{47}$

Protein Expression and Purification. The expression, isolation, and purification of WT and mutant HIV-1 proteases used for all assays and crystallization were carried out as previously described. ${ }^{46,48}$ Briefly, the gene encoding the desired HIV protease was subcloned into the heat-inducible pXC35 expression vector (ATCC) and transformed into E. coli TAP-106 cells. Cells grown in $6 \mathrm{~L}$ of Terrific Broth were lysed with a cell disruptor twice, and the protein was purified from inclusion bodies. ${ }^{49}$ The inclusion body centrifugation pellet was dissolved in 50\% acetic acid followed by another round of centrifugation at $19000 \mathrm{rpm}$ for $30 \mathrm{~min}$ to remove impurities. Size exclusion chromatography was carried out on a 2.1-L Sephadex G-75 superfine (Sigma Chemical) column equilibrated with $50 \%$ acetic acid to separate high molecular weight proteins from the desired protease. Pure fractions of HIV protease were refolded into a 10 -fold dilution of refolding buffer [0.05 $\mathrm{M}$ sodium acetate at $\mathrm{pH} 5.5,5 \%$ ethylene glycol, $10 \%$ glycerol, and $5 \mathrm{mM}$ DTT]. Folded protein was concentrated down to $0.5-3 \mathrm{mg} \mathrm{mL}^{-1}$ and stored. The stored protease was used in $K_{\mathrm{M}}$ and $K_{\mathrm{i}}$ assays. For crystallography, an additional purification step was performed with a Pharmacia Superdex 75 FPLC column equilibrated with refolding buffer. Protease fractions purified from the size exclusion column were concentrated to $1-2 \mathrm{mg} \mathrm{mL}^{-1}$ using an Amicon Ultra-15 10-kDa device (Millipore) for crystallization.

Enzyme Kinetics and Inhibition. $K_{m}$ Assay. $K_{\mathrm{m}}$ values were determined as previously described. ${ }^{22,37,50}$ Briefly, a 10 -amino acid substrate containing the natural $\mathrm{MA} / \mathrm{CA}$ cleavage site with an EDANS/DABCYL FRET pair was dissolved in $8 \%$ DMSO at $40 \mathrm{nM}$ and $6 \% \mathrm{DMSO}$ at $30 \mathrm{nM}$. The $30 \mathrm{nM}$ substrate was $4 / 5$ serially diluted from $30 \mathrm{nM}$ to $6 \mathrm{nM}$. HIV protease was diluted to $120 \mathrm{nM}$, and $5 \mu \mathrm{L}$ was added to the 96-well plate to obtain a final concentration of $10 \mathrm{nM}$. Fluorescence was observed with an excitation at $340 \mathrm{~nm}$ and emission at $492 \mathrm{~nm}$ and monitored for 200 counts, using a PerkinElmer Envision plate reader. A FRET inner filter effect correction was applied as previously described. ${ }^{51}$ Data corrected for the inner filter effect was analyzed with Prism7.

$K_{i}$ Assay. Enzyme inhibition constants $\left(K_{\mathrm{i}}\right.$ values) were determined as previously described. ${ }^{22,37,50}$ Briefly, in a 96-well plate, DRV was $3 / 5$ serially diluted from $2000 \mathrm{nM}$ for $8 \mathrm{Mut}, 1000 \mathrm{nM}$ for 9 Mut-A71V, $5000 \mathrm{nM}$ for 9Mut-L76V, $5000 \mathrm{nM}$ for 10 Mut, or $10000 \mathrm{nM}$ for 11 Mut. All samples were incubated with $5 \mathrm{nM}$ protein for $1 \mathrm{~h}$. A 10amino acid substrate containing an optimized protease cleavage site, ${ }^{37}$ purchased from Bachem, with an EDANS/DABCYL FRET pair was dissolved in 4\% DMSO at $120 \mathrm{mM}$. Using a PerkinElmer Envision plate reader, $5 \mu \mathrm{L}$ of the $120 \mathrm{mM}$ substrate was added to the 96-well plate to a final concentration of $10 \mathrm{mM}$. Fluorescence was observed with an excitation at $340 \mathrm{~nm}$ and emission at $492 \mathrm{~nm}$ and monitored for 200 counts. Data was analyzed with Prism7.

Crystal Structure Determination and Analysis. Crystallography. Discovery of the condition producing hexagonal cocrystals of DRV bound to a highly mutated variant (8Mut) without seeding was achieved using the Protein Complex Suite Screen (Qiagen), in well G5, consisting of $1 \mathrm{M}$ ammonium sulfate and $1 \mathrm{M}$ potassium chloride and 0.1 M HEPES buffer at $\mathrm{pH} 7$ with a protease concentration of 1.2 $\mathrm{mg} \mathrm{mL}^{-1}$ and 5-fold molar excess of DRV. With previously generated orthorhombic and hexagonal cocrystals in hand, reproducible cocrystals of DRV bound to all variants were achieved as previously described. ${ }^{22}$ Briefly, all cocrystals were grown at RT by hanging drop vapor diffusion method in 24-well VDX hanging-drop trays (Hampton Research) with protease concentrations between 1.0 and $2.4 \mathrm{mg} \mathrm{mL} \mathrm{mi}^{-1}$ with 3-, 5-, or 10-fold molar excess of DRV. Crystallization drops were set with the reservoir solution consisting of $18-26 \%(\mathrm{w} / \mathrm{v})$ ammonium sulfate and $0.1 \mathrm{M}$ bis-Tris-methane buffer at $\mathrm{pH} 5.5$ set with $2 \mu \mathrm{L}$ of well solution and $1 \mu \mathrm{L}$ protein and microseeded with a cat whisker. Crystal morphology and space group was entirely dependent on the microseeds. Diffraction quality crystals were obtained within 1 week. As data was collected at $100 \mathrm{~K}$, cryogenic conditions contained the precipitant solution supplemented with $25 \%$ glycerol. For direct structural analysis without possible crystal lattice contact bias, structure of WT protease bound to DRV was also generated and determined in the hexagonal space group.

Data Collection and Structure Solution. Diffraction data were collected and solved as previously described. ${ }^{22,30,46}$ Diffraction quality crystals were flash frozen under a cryostream when mounting the crystal at our in-house Rigaku Saturn944 X-ray system. The data for 10Mut cocrystal structure were collected at the Advanced Photon Source at the Argonne National Laboratory, beamline 19-ID. All cocrystal diffraction intensities were indexed, integrated, and scaled using HKL3000. ${ }^{52}$ Structures were solved using molecular replacement with PHASER. ${ }^{53}$ Model building and refinement were performed using Coot ${ }^{54}$ and Phenix. ${ }^{55}$ During refinement, all crystals utilized optimized stereochemical weights and noncrystallographic symmetry operators. Hexagonal crystals grew as pseudomerohedral twins and were solved with a twin law applied (h,-h-k,-l). Ligands were designed in Maestro, and the output sdf file was used in the Phenix program eLBOW ${ }^{56}$ to generate the cif file containing atomic positions and constraints necessary for ligand refinement. Iterative rounds of crystallographic refinement were carried out until convergence was achieved. To limit bias throughout the refinement process, 5\% of the data were reserved for the free R-value calculation. ${ }^{57}$ MolProbity ${ }^{58}$ was applied to evaluate the final structures before deposition in the PDB. Structure analysis, superposition, and figure generation were performed using PyMOL. ${ }^{59} \mathrm{X}$-ray data collection and crystallographic refinement statistics are presented in the Supporting Information [Table S2].

Internal Distance Analysis of Crystal Structures. Distancedifference matrices were generated as previously described ${ }^{60}$ to reveal structural changes between inhibitor-protease pairs.

Intermolecular vdW Contact Analysis of Crystal Structures. To calculate the intermolecular van der Waals (vdW) interaction energies the crystal structures were prepared using the Schrodinger Protein Preparation Wizard. ${ }^{61}$ Hydrogen atoms were added, protonation states were determined, and the structures were minimized. The protease active site was monoprotonated at D25. Subsequently, force field parameters were assigned using the OPLS2005 force field. ${ }^{62}$ Interaction energies between the inhibitor and protease were estimated using a simplified Lennard-Jones potential, as previously described in detail. ${ }^{63}$ Briefly, the vdW energy was calculated for pairwise interactions depending on the types of atoms interacting and the distance between them. For each protease residue, the change in $\mathrm{vdW}$ interactions relative to a WT complex in the same space group was also calculated for each variant structure.

Molecular Dynamics Simulations and Analysis. System Preparation. High-resolution crystal structures were prepared using the Protein Preparation Wizard from Maestro within the Schrodinger Suite $^{61}$ as previously described. ${ }^{64}$ Briefly, cocrystallized phosphates were removed, missing atoms were added using Prime, ${ }^{65}$ and PROPKA $^{66,67}$ was used to determine the protonation state of side chains at $\mathrm{pH}$ 7.0. Lastly, the structure was minimized to a convergence criterion of $0.3 \AA$ using Impref. ${ }^{68}$

Molecular Dynamics Simulations. The prepared systems were placed in a cubic TIP3P water box measuring $12 \AA$ on each side. MD simulations were carried out as previously described ${ }^{64}$ using Desmond software suite within Schrodinger. ${ }^{69}$ Briefly, chloride ions were used to neutralize the system, and $0.15 \mathrm{M}$ salt was added using sodium and chloride ions. The OPLS3 force field was used to parametrize the ligand and protein. Prior to starting the $100 \mathrm{~ns}$ MD simulations, the solvated system was minimized using the stepwise procedure described previously. ${ }^{64}$ Triplicates of $100 \mathrm{~ns}$ simulations for WT, I84V, 2Mut, 4Mut, 8Mut, 9Mut-A71V, 9Mut-L76V, 10Mut, and 11 Mut each with a randomized velocity were started using the protocol previously developed. ${ }^{64}$ The root-mean-square deviation (RMSD) and root-mean-square fluctuation (RMSF) of $\mathrm{C}_{\alpha}$ atoms were calculated by utilizing the simulation interactions diagram from Maestro within the Schrodinger Suite.

Intermolecular vdW Contact Analysis of MD Trajectories. Intermolecular vdW interactions were calculated using a previously 
published protocol utilizing Lennard-Jones potential. ${ }^{46,70}$ The force field is not optimized for a sulfur violating the octet rule such as that found in the P2' moiety of DRV. As such, the packing around the P2' sulfur was calculated by averaging the vdW packing of the four adjacent atoms.

Dynamic Structural Analysis. Cross-Correlations of Protease/ DRV Fluctuations. The cross-correlation coefficients between the protease $\mathrm{C}_{\alpha}$ atoms and DRV heavy atoms were calculated using a previously published protocol. ${ }^{28}$ Briefly, the atom fluctuations were determined for each MD simulation. The cross-correlation between atom pairs, such as atoms $i$ and $j$, was determined according to

$$
C_{i, j}=\frac{\left\langle\Delta R_{i} \Delta R_{j}\right\rangle}{\sqrt{\left\langle\Delta R_{i}{ }^{2}\right\rangle\left\langle\Delta R_{j}^{2}\right\rangle}}
$$

where $\Delta R_{i}$ and $\Delta R_{j}$ are the positional changes of atoms $i$ and $j$, respectively, and the angle brackets denote an ensemble average. The cross-correlation coefficient ranges from -1 to 1 , where -1 represents complete anticorrelation between the atom pair, zero represents no correlation, and 1 represents complete correlation. The crosscorrelation values were calculated using an in-house script and mapped onto the protease structure by replacing the B-factors in the PDB coordinate file, using PyMOL. ${ }^{59}$

Distance Difference Matrices. The distances between all $\mathrm{C}_{\alpha}$ atom pairs for a given structure were calculated as a $198 \times 198$ matrix for all frames in the trajectory. The distance was calculated for each $\mathrm{C}_{\alpha}$ atom pair, giving the mean distance over the trajectory. The distance difference matrix was produced by subtracting each mean distance in the reference structure from the corresponding mean distance in the given structure. The overall difference for each residue was then calculated by taking the average of the absolute values of all 198 distance differences involving that residue. $\mathrm{PyMOL}^{59}$ was used for visualization of distance differences where the protease backbone was represented as a cartoon-putty with increasing thickness and warmer color for increasing deviation.

Jensen-Shannon Divergence Analysis of Dihedral Angles. Over the MD simulations, all the $\phi, \psi$, and $\chi$ dihedral angles of protease residues were measured. These dihedral angle measurements were used as input for the MutInf software package. ${ }^{45}$ Utilizing this package, the Jensen-Shannon divergence (JSD), which analyzes the difference between two probability distributions, was calculated. An $\alpha$ of 0.05 was used as a threshold to filter out nonstatistically significant differences in distributions, where a $p$-value $>\alpha$ was considered nonstatistically significant. In such cases, the JSD was set to zero. For visualization purposes, using PyMOL, ${ }^{59}$ if a residue had multiple dihedral angles with a calculated JSD, the greatest value was selected.

\section{ASSOCIATED CONTENT}

\section{S Supporting Information}

The Supporting Information is available free of charge on the ACS Publications website at DOI: 10.1021/acschembio.9b00370.

Additional enzyme kinetics, structural, and dynamics data and X-ray crystallography statistics (PDF)

\section{AUTHOR INFORMATION}

\section{Corresponding Authors}

*C.A.S. E-mail: Celia.Schiffer@umassmed.edu.

*N.K.Y. E-mail: Nese.KurtYilmaz@umassmed.edu.

ORCID $\odot$

Gordon J. Lockbaum: 0000-0003-2720-6984

Nese Kurt Yilmaz: 0000-0002-5036-676X

Celia A. Schiffer: 0000-0003-2270-6613

\section{Author Contributions}

${ }^{\S}$ M.H. and G.J.L. are joint first authors.

\section{Notes}

The authors declare no competing financial interest.

\section{ACKNOWLEDGMENTS}

This research was supported by National Institute of General Medical Sciences Grant P01 GM109767. Some of the crystallographic data were collected at Argonne National Laboratory, Structural Biology Center (SBC) at the Advanced Photon Source. SBC-CAT is operated by UChicago Argonne, LLC, for the U.S. Department of Energy, Office of Biological and Environmental Research under Contract DE-AC0206CH11357. We thank the beamline specialists at 19-ID for their help in data collection.

\section{REFERENCES}

(1) O’Neill, J. Antimicrobial Resistance: Tackling a crisis for the health and wealth of nations. http://amr-review.org/sites/default/ file s / A M R 20 R e vi e w $\% 20$ P a p e r \% 20 $\% 20$ Tackling $\% 20 \mathrm{a} \% 20$ crisis $\% 20$ for $\% 20$ the $\% 20$ health $\% 20$ and $\% 20$ wealth\%20of\%20nations_1.pdf (accessed April12, 2019).

(2) The PLOS Medicine Editors (2016) Antimicrobial Resistance: Is the World UNprepared? PLoS Med1 3, No. e1002130.

(3) World Health Organization. (2018) Fact Sheet: Antimicrobial Resistance. https://www.who.int/en/news-room/fact-sheets/detail/ antimicrobial-resistance.

(4) Galvani, A. P. (2005) The role of mutation accumulation in HIV progression. Proc. R. Soc. London, Ser. B 272, 1851-1858.

(5) Maldarelli, F., Kearney, M., Palmer, S., Stephens, R., Mican, J., Polis, M. A., Davey, R. T., Kovacs, J., Shao, W., Rock-Kress, D., Metcalf, J. A., Rehm, C., Greer, S. E., Lucey, D. L., Danley, K., Alter, H., Mellors, J. W., and Coffin, J. M. (2013) HIV populations are large and accumulate high genetic diversity in a nonlinear fashion. J. Virol 87, 10313-10323.

(6) Patel, P. H., and Preston, B. D. (1994) Marked infidelity of human immunodeficiency virus type 1 reverse transcriptase at RNA and DNA template ends. Proc. Natl. Acad. Sci. U. S. A. 91, 549-553.

(7) Abram, M. E., Ferris, A. L., Das, K., Quinones, O., Shao, W., Tuske, S., Alvord, W. G., Arnold, E., and Hughes, S. H. (2014) Mutations in HIV-1 reverse transcriptase affect the errors made in a single cycle of viral replication. J. Virol 88, 7589-7601.

(8) Abram, M. E., Ferris, A. L., Shao, W., Alvord, W. G., and Hughes, S. H. (2010) Nature, position, and frequency of mutations made in a single cycle of HIV-1 replication. J. Virol 84, 9864-9878.

(9) Smyth, R. P., Schlub, T. E., Grimm, A. J., Waugh, C., Ellenberg, P., Chopra, A., Mallal, S., Cromer, D., Mak, J., Davenport, M. P., and Hahn, B. H. (2014) Identifying recombination hot spots in the HIV-1 genome. J. Virol 88, 2891-2902.

(10) Onafuwa-Nuga, A., and Telesnitsky, A. (2009) The remarkable frequency of human immunodeficiency virus type 1 genetic recombination. Microbiol Mol. Biol. Rev. 73, 451-480.

(11) Yasuda, J. M., Miller, C., Currier, J. S., Forthal, D. N., Kemper, C. A., Beall, G. N., Tilles, J. G., Capparelli, E. V., McCutchan, J. A., Haubrich, R. H., and Treatment, G. (2004) The correlation between plasma concentrations of protease inhibitors, medication adherence and virological outcome in HIV-infected patients. Antivir Ther 9, 753-761 and California Collaborative .

(12) Wu, T. D., Schiffer, C. A., Gonzales, M. J., Taylor, J., Kantor, R., Chou, S., Israelski, D., Zolopa, A. R., Fessel, W. J., and Shafer, R. W. (2003) Mutation patterns and structural correlates in human immunodeficiency virus type 1 protease following different protease inhibitor treatments. J. Virol 77, 4836-4847.

(13) Clavel, F., and Mammano, F. (2010) Role of Gag in HIV Resistance to Protease Inhibitors. Viruses 2, 1411-1426.

(14) Dam, E., Quercia, R., Glass, B., Descamps, D., Launay, O., Duval, X., Krausslich, H. G., Hance, A. J., Clavel, F., and ANRS 109 Study Group (2009) Gag mutations strongly contribute to HIV-1 
resistance to protease inhibitors in highly drug-experienced patients besides compensating for fitness loss. PLoS Pathog. 5, No. e1000345. (15) Surleraux, D. L., Tahri, A., Verschueren, W. G., Pille, G. M., de Kock, H. A., Jonckers, T. H., Peeters, A., De Meyer, S., Azijn, H., Pauwels, R., de Bethune, M. P., King, N. M., Prabu-Jeyabalan, M., Schiffer, C. A., and Wigerinck, P. B. (2005) Discovery and selection of TMC114, a next generation HIV-1 protease inhibitor. J. Med. Chem. $48,1813-1822$

(16) Nalam, M. N., Peeters, A., Jonckers, T. H., Dierynck, I., and Schiffer, C. A. (2007) Crystal structure of lysine sulfonamide inhibitor reveals the displacement of the conserved flap water molecule in human immunodeficiency virus type 1 protease. J. Virol 81, 95129518.

(17) Cai, Y., and Schiffer, C. A. (2010) Decomposing the energetic impact of drug resistant mutations in HIV-1 protease on binding DRV. J. Chem. Theory Comput. 6, 1358-1368.

(18) Prabu-Jeyabalan, M., Nalivaika, E., and Schiffer, C. A. (2002) Substrate shape determines specificity of recognition for HIV-1 protease: analysis of crystal structures of six substrate complexes. Structure 10, 369-381.

(19) Ali, A., Bandaranayake, R. M., Cai, Y., King, N. M., Kolli, M., Mittal, S., Murzycki, J. F., Nalam, M. N., Nalivaika, E. A., Ozen, A., Prabu-Jeyabalan, M. M., Thayer, K., and Schiffer, C. A. (2010) Molecular Basis for Drug Resistance in HIV-1 Protease. Viruses 2, $2509-2535$.

(20) Lv, Z., Chu, Y., and Wang, Y. (2015) HIV protease inhibitors: a review of molecular selectivity and toxicity. HIV/AIDS 7, 95-104.

(21) Nalam, M. N., Ali, A., Reddy, G. S., Cao, H., Anjum, S. G., Altman, M. D., Yilmaz, N. K., Tidor, B., Rana, T. M., and Schiffer, C. A. (2013) Substrate envelope-designed potent HIV-1 protease inhibitors to avoid drug resistance. Chem. Biol. 20, 1116-1124.

(22) Lockbaum, G. J., Leidner, F., Rusere, L. N., Henes, M., Kosovrasti, K., Nachum, G. S., Nalivaika, E. A., Ali, A., Yilmaz, N. K., and Schiffer, C. A. (2019) Structural Adaptation of Darunavir Analogues against Primary Mutations in HIV-1 Protease. ACS Infect. Dis. 5, 316-325.

(23) Wensing, A. M., van Maarseveen, N. M., and Nijhuis, M. (2010) Fifteen years of HIV Protease Inhibitors: raising the barrier to resistance. Antiviral Res. 85, 59-74.

(24) Muzammil, S., Ross, P., and Freire, E. (2003) A major role for a set of non-active site mutations in the development of HIV-1 protease drug resistance. Biochemistry 42, 631-638.

(25) Ohtaka, H., Schon, A., and Freire, E. (2003) Multidrug resistance to HIV-1 protease inhibition requires cooperative coupling between distal mutations. Biochemistry 42, 13659-13666.

(26) Clemente, J. C., Moose, R. E., Hemrajani, R., Whitford, L. R., Govindasamy, L., Reutzel, R., McKenna, R., Agbandje-McKenna, M., Goodenow, M. M., and Dunn, B. M. (2004) Comparing the accumulation of active- and nonactive-site mutations in the HIV-1 protease. Biochemistry 43, 12141-12151.

(27) Prachanronarong, K. L., Canale, A. S., Liu, P., Somasundaran, M., Hou, S., Poh, Y.-P., Han, T., Zhu, Q., Renzette, N., Zeldovich, K. B., Kowalik, T. F., Kurt-Yilmaz, N., Jensen, J. D., Bolon, D. N. A., Marasco, W. A., Finberg, R. W., Schiffer, C. A., and Wang, J. P. (2019) Mutations in Influenza A Virus Neuraminidase and Hemagglutinin Confer Resistance against a Broadly Neutralizing Hemagglutinin Stem Antibody. J. Virol. 93, No. e01639-18.

(28) Paulsen, J. L., Leidner, F., Ragland, D. A., Kurt Yilmaz, N., and Schiffer, C. A. (2017) Interdependence of Inhibitor Recognition in HIV-1 Protease. J. Chem. Theory Comput. 13, 2300-2309.

(29) Ragland, D. A., Nalivaika, E. A., Nalam, M. N., Prachanronarong, K. L., Cao, H., Bandaranayake, R. M., Cai, Y., Kurt-Yilmaz, N., and Schiffer, C. A. (2014) Drug resistance conferred by mutations outside the active site through alterations in the dynamic and structural ensemble of HIV-1 protease. J. Am. Chem. Soc. 136, 11956-11963.

(30) Soumana, D. I., Kurt Yilmaz, N., Ali, A., Prachanronarong, K. L., and Schiffer, C. A. (2016) Molecular and Dynamic Mechanism
Underlying Drug Resistance in Genotype 3 Hepatitis C NS3/4A Protease. J. Am. Chem. Soc. 138, 11850-11859.

(31) Matthew, A. N., Leidner, F., Newton, A., Petropoulos, C. J., Huang, W., Ali, A., KurtYilmaz, N., and Schiffer, C. A. (2018) Molecular Mechanism of Resistance in a Clinically Significant Double-Mutant Variant of HCV NS3/4A Protease. Structure 26, 1360-1372.

(32) Rhee, S. Y., Gonzales, M. J., Kantor, R., Betts, B. J., Ravela, J., and Shafer, R. W. (2003) Human immunodeficiency virus reverse transcriptase and protease sequence database. Nucleic Acids Res. 31, 298-303.

(33) Shafer, R. W. (2006) Rationale and uses of a public HIV drugresistance database. J. Infect. Dis. 194, S51-58.

(34) Ragland, D. A., Whitfield, T. W., Lee, S. K., Swanstrom, R., Zeldovich, K. B., Kurt-Yilmaz, N., and Schiffer, C. A. (2017) Elucidating the Interdependence of Drug Resistance from Combinations of Mutations. J. Chem. Theory Comput. 13, 5671-5682.

(35) Lee, S. K., Potempa, M., Kolli, M., Ozen, A., Schiffer, C. A., and Swanstrom, R. (2012) Context surrounding processing sites is crucial in determining cleavage rate of a subset of processing sites in HIV-1 Gag and Gag-Pro-Pol polyprotein precursors by viral protease. J. Biol. Chem. 287, 13279-13290.

(36) Deshmukh, L., Louis, J. M., Ghirlando, R., and Clore, G. M. (2016) Transient HIV-1 Gag-protease interactions revealed by paramagnetic NMR suggest origins of compensatory drug resistance mutations. Proc. Natl. Acad. Sci. U. S. A. 113, 12456-12461.

(37) Windsor, I. W., and Raines, R. T. (2015) Fluorogenic Assay for Inhibitors of HIV-1 Protease with Sub-picomolar Affinity. Sci. Rep. 5, 11286.

(38) King, N. M., Prabu-Jeyabalan, M., Nalivaika, E. A., Wigerinck, P., de Bethune, M. P., and Schiffer, C. A. (2004) Structural and thermodynamic basis for the binding of TMC114, a next-generation human immunodeficiency virus type 1 protease inhibitor. J. Virol 78, 12012-12021.

(39) Horovitz, A. (1996) Double-mutant cycles: a powerful tool for analyzing protein structure and function. Folding Des. 1, R121-126.

(40) Goldfarb, N. E., Ohanessian, M., Biswas, S., McGee, T. D., Jr., Mahon, B. P., Ostrov, D. A., Garcia, J., Tang, Y., McKenna, R., Roitberg, A., and Dunn, B. M. (2015) Defective hydrophobic sliding mechanism and active site expansion in HIV-1 protease drug resistant variant Gly48Thr/Leu89Met: mechanisms for the loss of saquinavir binding potency. Biochemistry 54, 422-433.

(41) Foulkes-Murzycki, J. E., Scott, W. R., and Schiffer, C. A. (2007) Hydrophobic sliding: a possible mechanism for drug resistance in human immunodeficiency virus type 1 protease. Structure 15, 225233.

(42) Ozen, A., Sherman, W., and Schiffer, C. A. (2013) Improving the Resistance Profile of Hepatitis C NS3/4A Inhibitors: Dynamic Substrate Envelope Guided Design. J. Chem. Theory Comput. 9, 56935705.

(43) Nijhuis, M., Schuurman, R., de Jong, D., Erickson, J., Gustchina, E., Albert, J., Schipper, P., Gulnik, S., and Boucher, C. A. (1999) Increased fitness of drug resistant HIV-1 protease as a result of acquisition of compensatory mutations during suboptimal therapy. AIDS 13, 2349-2359.

(44) Meher, B. R., and Wang, Y. (2012) Interaction of I50V mutant and I50L/A71V double mutant HIV-protease with inhibitor TMC114 (darunavir): molecular dynamics simulation and binding free energy studies. J. Phys. Chem. B 116, 1884-1900.

(45) McClendon, C. L., Hua, L., Barreiro, A., and Jacobson, M. P. (2012) Comparing Conformational Ensembles Using the KullbackLeibler Divergence Expansion. J. Chem. Theory Comput. 8, 21152126.

(46) Ozen, A., Lin, K. H., Kurt Yilmaz, N., and Schiffer, C. A. (2014) Structural basis and distal effects of Gag substrate coevolution in drug resistance to HIV-1 protease. Proc. Natl. Acad. Sci. U. S. A. 111, 15993-15998. 
(47) Rose, J. R., Salto, R., and Craik, C. S. (1993) Regulation of autoproteolysis of the HIV-1 and HIV-2 proteases with engineered amino acid substitutions. J. Biol. Chem. 268, 11939-11945.

(48) King, N. M., Melnick, L., Prabu-Jeyabalan, M., Nalivaika, E. A., Yang, S. S., Gao, Y., Nie, X., Zepp, C., Heefner, D. L., and Schiffer, C. A. (2002) Lack of synergy for inhibitors targeting a multi-drugresistant HIV-1 protease. Protein Sci. 11, 418-429.

(49) Hui, J. O., Tomasselli, A. G., Reardon, I. M., Lull, J. M., Brunner, D. P., Tomich, C.-S. C., and Heinrikson, R. L. (1993) Large scale purification and refolding of HIV-1 protease fromEscherichia coli inclusion bodies. J. Protein Chem. 12, 323-327.

(50) Matayoshi, E. D., Wang, G. T., Krafft, G. A., and Erickson, J. (1990) Novel fluorogenic substrates for assaying retroviral proteases by resonance energy transfer. Science 247, 954-958.

(51) Liu, Y., Kati, W., Chen, C. M., Tripathi, R., Molla, A., and Kohlbrenner, W. (1999) Use of a fluorescence plate reader for measuring kinetic parameters with inner filter effect correction. Anal. Biochem. 267, 331-335.

(52) Otwinowski, Z., and Minor, W. (1997) [20] Processing of Xray diffraction data collected in oscillation mode. Methods Enzymol. 276, 307-326.

(53) McCoy, A. J., Grosse-Kunstleve, R. W., Adams, P. D., Winn, M. D., Storoni, L. C., and Read, R. J. (2007) Phaser crystallographic software. J. Appl. Crystallogr. 40, 658-674.

(54) Emsley, P., and Cowtan, K. (2004) Coot: model-building tools for molecular graphics. Acta Crystallogr., Sect. D: Biol. Crystallogr. 60, 2126-2132.

(55) Adams, P. D., Afonine, P. V., Bunkoczi, G., Chen, V. B., Davis, I. W., Echols, N., Headd, J. J., Hung, L. W., Kapral, G. J., GrosseKunstleve, R. W., McCoy, A. J., Moriarty, N. W., Oeffner, R., Read, R. J., Richardson, D. C., Richardson, J. S., Terwilliger, T. C., and Zwart, P. H. (2010) PHENIX: a comprehensive Python-based system for macromolecular structure solution. Acta Crystallogr., Sect. D: Biol. Crystallogr. 66, 213-221.

(56) Moriarty, N. W., Grosse-Kunstleve, R. W., and Adams, P. D. (2009) electronic Ligand Builder and Optimization Workbench (eLBOW): a tool for ligand coordinate and restraint generation. Acta Crystallogr., Sect. D: Biol. Crystallogr. 65, 1074-1080.

(57) Brunger, A. T. (1992) Free R value: a novel statistical quantity for assessing the accuracy of crystal structures. Nature 355, 472-475.

(58) Davis, I. W., Leaver-Fay, A., Chen, V. B., Block, J. N., Kapral, G. J., Wang, X., Murray, L. W., Arendall, W. B., 3rd, Snoeyink, J., Richardson, J. S., and Richardson, D. C. (2007) MolProbity: all-atom contacts and structure validation for proteins and nucleic acids. Nucleic Acids Res. 35, W375-383.

(59) PyMOL Molecular Graphics System, version 2.0; Schrödinger, LLC

(60) Prabu-Jeyabalan, M., Nalivaika, E. A., Romano, K., and Schiffer, C. A. (2006) Mechanism of substrate recognition by drug-resistant human immunodeficiency virus type 1 protease variants revealed by a novel structural intermediate. J. Virol 80, 3607-3616.

(61) Madhavi Sastry, G., Adzhigirey, M., Day, T., Annabhimoju, R., and Sherman, W. (2013) Protein and ligand preparation: parameters, protocols, and influence on virtual screening enrichments. J. Comput.Aided Mol. Des. 27, 221-234.

(62) Jorgensen, W. L., Maxwell, D. S., and Tirado-Rives, J. (1996) Development and testing of the OPLS all-atom force field on conformational energetics and properties of organic liquids. J. Am. Chem. Soc. 118, 11225-11236.

(63) Nalam, M. N., Ali, A., Altman, M. D., Reddy, G. S., Chellappan, S., Kairys, V., Ozen, A., Cao, H., Gilson, M. K., Tidor, B., Rana, T. M., and Schiffer, C. A. (2010) Evaluating the substrate-envelope hypothesis: structural analysis of novel HIV-1 protease inhibitors designed to be robust against drug resistance. J. Virol 84, 5368-5378.

(64) Leidner, F., Kurt Yilmaz, N., Paulsen, J., Muller, Y. A., and Schiffer, C. A. (2018) Hydration Structure and Dynamics of Inhibitor-Bound HIV-1 Protease. J. Chem. Theory Comput. 14, 2784-2796.
(65) Jacobson, M. P., Friesner, R. A., Xiang, Z., and Honig, B. (2002) On the role of the crystal environment in determining protein side-chain conformations. J. Mol. Biol. 320, 597-608.

(66) Olsson, M. H., Sondergaard, C. R., Rostkowski, M., and Jensen, J. H. (2011) PROPKA3: Consistent Treatment of Internal and Surface Residues in Empirical pKa Predictions. J. Chem. Theory Comput. 7, 525-537.

(67) Sondergaard, C. R., Olsson, M. H., Rostkowski, M., and Jensen, J. H. (2011) Improved Treatment of Ligands and Coupling Effects in Empirical Calculation and Rationalization of $\mathrm{pKa}$ Values. J. Chem. Theory Comput. 7, 2284-2295.

(68) Banks, J. L., Beard, H. S., Cao, Y., Cho, A. E., Damm, W., Farid, R., Felts, A. K., Halgren, T. A., Mainz, D. T., Maple, J. R., Murphy, R., Philipp, D. M., Repasky, M. P., Zhang, L. Y., Berne, B. J., Friesner, R. A., Gallicchio, E., and Levy, R. M. (2005) Integrated Modeling Program, Applied Chemical Theory (IMPACT). J. Comput. Chem. 26, $1752-1780$

(69) Bowers, K. J., Chow, D. E., Xu, H., Dror, R. O., Eastwood, M. P., Gregersen, B. A., Klepeis, J. L., Kolossvary, I., Moraes, M. A., Sacerdoti, F. D., Salmon, J. K., Shan, Y., and Shaw, D. E. (2006) Scalable Algorithms for Molecular Dynamics Simulations on Commodity Clusters. SC 2006: Proceedings Supercomputing, 43-43.

(70) Ozen, A., Haliloglu, T., and Schiffer, C. A. (2011) Dynamics of preferential substrate recognition in HIV-1 protease: redefining the substrate envelope. J. Mol. Biol. 410, 726-744. 